\title{
A rare case of deep vein and right atrial thrombosis in a patient with chronic heart failure and pulmonary embolism
}

\author{
Akhmetzhan Sugraliyev ${ }^{1}$, Shynar Aktayeva ${ }^{2}$, Gulnur Tanbayeva ${ }^{2}$, Almat Kodasbayev ${ }^{1}$, Plinio Cirillo ${ }^{3}$, \\ Klara Iskakova ${ }^{2}$ \\ ${ }^{1}$ Asfendiyarov Kazakh National Medical University, Almaty, Kazakhstan; ${ }^{2} J S C$ "Central Clinical Hospital”, Almaty, \\ Kazakhstan; ${ }^{3}$ Department of Advanced Biomedical Sciences, University of Naples Federico II, Italy
}

\begin{abstract}
Deep vein thrombosis (DVT) is frequently observed in patients with chronic heart failure (CHF), increasing the risk of pulmonary embolism (PE). Clinical evaluation of CHF patients with suspected acute $\mathrm{PE}$ is challenging since these diseases share several symptoms and signs such as dyspnea. Thus, it is intuitive that correct and fast diagnosis of PE in these patients might be able to significantly change their clinical outcome. In the present report, we describe a rare case of a patient with $\mathrm{CHF}$ and $\mathrm{PE}$ due to a huge thrombosis of deep veins and of right atrium in whom echo evaluation permitted the correct diagnosis and therapy.
\end{abstract}

\section{Introduction}

Deep venous thrombosis (DVT) is frequently observed in patients with chronic heart failure (CHF), ranging from $1 \%$ to $59 \%$

Correspondence: Akhmetzhan Sugraliyev, Department of Internal Diseases, Asfendiyarov Kazakh National Medical University, 94 Tole bi str., 050000 Almaty, Kazakhstan. E- mail: asugraliyev@gmail.com

Key words: Chronic heart failure; thrombosis; pulmonary embolism.

Conflict of interest: The authors declare no conflict of interest.

Contributions: All the Authors contributed equally. All the authors have read and approved the final version of the manuscript and agreed to be accountable for all aspects of the work.

Patient consent for publication: Written informed consent was obtained from the patient for the publication of this study.

Received for publication: 5 August 2019

Accepted for publication: 18 December 2019.

${ }^{\circ}$ Copyright: the Author(s), 2020

Licensee PAGEPress, Italy

Monaldi Archives for Chest Disease 2020; 90:1142

doi: 10.4081/monaldi.2020.1142

This article is distributed under the terms of the Creative Commons Attribution Noncommercial License (by-nc 4.0) which permits any noncommercial use, distribution, and reproduction in any medium, provided the original author(s) and source are credited.
$[1,2]$. This variability might be due to the different sensitivity of diagnostic criteria in reports: those hospitals, in which the screening for DVT is more accurate, are likely to find more cases of DVT and pulmonary embolism (PE) [3]. Myocardial infarction and heart failure increase the risk of PE. Conversely, patients with DVT have an increased risk of developing myocardial infarction and stroke [4]. Thus, a correct and fast diagnosis of PE in these patients plays a pivotal role to change their clinical outcome.

In the present report, we describe the case of a patient with $\mathrm{CHF}$ complicated by PE due to a huge venous thrombosis that extended from pheripheral vein till the right atrium, where echocardiographic evaluation showed a rare image of thrombus floating in the cavity of cardiac chamber.

\section{Case Report}

A 69-year-old male patient, heavy smoker, with two years clinical history of chronic heart failure (CHF) after a myocardial infarction and with chronic obstructive lung disease (COPD) was admitted at emergency department because he had cough, dyspnea at rest, exacerbated by physical exertion, orthopnea and poor appetite. Clinical parameters and blood sample values on admission are reported in Table 1. Scattered wheezing at bilateral lung, large edema of lower extremities more extensive on the left side and varicose veins of lower extremities to the level of the lower third of the hips prominent in the left leg were observed. Chest x-ray (CXR) in PA views: normal; ECG showed sinus tachycardia with signs of old infarction in inferior wall. The patient was transferred to intensive care unit (ICU) where a significant increase of NTproBNP and of creatinine levels and reduced values of eGFR were observed (Table 1). Echocardiogram showed dilated left ventricle (LVDd: $66 \mathrm{~mm}$; LVDs: $59 \mathrm{~mm}$ ), dilated right ventricle (RVD: 45 $\mathrm{mm})$ and right atrium $(55 \mathrm{~mm})$, diffuse left ventricular hypokinesia, severe LVEF reduction (20-22\%; Simpson). TAPSE: $13 \mathrm{~mm}$, increased pulmonary pressure $(55 \mathrm{mmHg})$. Interestingly, in the right atrial cavity, close to the entering of the vena cava inferior (VCI), a thrombus formation was detected. The risk of DVT and PE was measured according to the Well's criteria. A score of 6 and 7.5 for DVT and PE respectively was measued, confirming DVT and PE diagnosis. Indeed, ultrasound examination of vein showed a huge thrombosis that started from the popliteal and femoral veins, continued in the iliac segment and ended in inferior vena cava (IVC) where a thrombus $(12 \times 114 \mathrm{~mm})$ moving with the blood flow was detected (free-floating inferior vena cava thrombosis, IVCT) (Figure $1 \mathrm{~A}, \mathrm{~B}$ ). In ICU the patient became unstable with persistent breathlessness, increasing of tachycardia, decreasing 

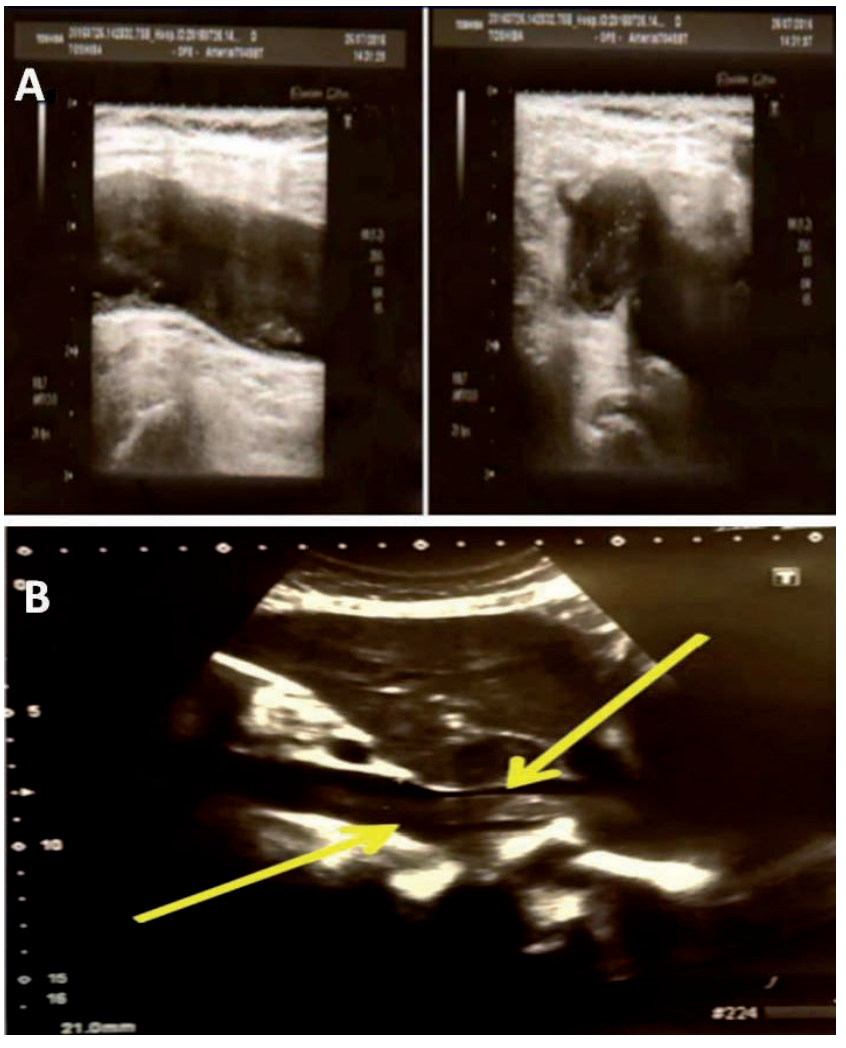

Figure 1. A) Ultrasound examination of lower extremities demonstrates extensive thrombosis starting from the popliteal and femoral veins and ending to the iliac segment. B) Ultrasound examination of inferior vena cava (IVC). Arrows indicate a thrombus $(12 \times 114 \mathrm{~mm}$ ) moving with the blood flow (free-floating inferior vena cava thrombosis, IVCT). blood pressure and oxygen saturation pO2 (Table 1) but without remarkable chest pain and ECG. At this timepoint, $5000 \mathrm{IU}$ followed by a drip of $1200 \mathrm{IU} / \mathrm{h}$ of unfractioned heparin (UFH) were administered to reach a target for activated partial thromboplastin time of 60 to 80 seconds and the thrombolityc option was considered only in case of worsening of clinical status. Twelve hours later the symptoms improved and three days later, once the patient had acceptable values of creatinine $(83 \mathrm{mmol} / \mathrm{L})$ and of eGFR (56 $\mathrm{mL} / \mathrm{min}$ ), MDCT was performed, showing segmental PE, PESI 2 Class. The patient was switched from to DOAC (Dabigatran 110 $\mathrm{mg}$ b.i.d) taking into account fact that clinical significance of MDCT angiography in case of isolated sub-segmental PE is poor.

Seven days later, echocardiogram showed complete thrombus resolution in the right atrial cavity. Moreover, ultrasound examination of vein showed the complete resolution of IVCT (Figure 2A]. On the contrary, thrombosis of the popliteal and deep femoral veins to the level of the femoral-iliac segments of left side was still observed (Figure 2B). After two weeks of hospitalization, plasma levels of NT-proBNP were significantly reduced from 24 $478 \mathrm{pg} / \mathrm{ml}$ to $9871 \mathrm{pg} / \mathrm{ml}$ and the patient was discharged on oral anticoagulant and specific CHF therapy (ramipril $2.5 \mathrm{mg}$ QD, target dose $5 \mathrm{mg}$ BID, Carvedilol 6.25 BID target dose 25-50 BID, spironolactone $25 \mathrm{mg}$ QD, ivabradine $5 \mathrm{mg}$ BID, Torasemide $10 \mathrm{mg}$ once per week and dabigatran $110 \mathrm{mg}$ BID.

After discharge, ultrasound examination scheduled at $2^{\text {nd }}$ month showed the absence of thrombosis (Figure 2C) and one month later, treatment with direct oral anticoagulants (DOACs) therapy was stopped and patient was switched to treatment with sulodexide on top of aspirin. After 1 year management with ACE inhibitors, betablockers, mineralocorticoid receptor antagonists and correction of iron deficiency, patient was stable with increased values of EF (42\%) and he did not have any other access to the ER.

Table 1. Laboratory data during in hospital stay and at 1 year follow up.

\begin{tabular}{|c|c|c|c|c|}
\hline & On admission & ICU & On discharge & After 1 year \\
\hline HR (bpm) & 88 & 98 & 82 & 72 \\
\hline $\mathrm{BP}(\mathrm{mmHg})$ & $110 / 65$ & $90 / 65$ & $105 / 65$ & $110 / 70$ \\
\hline SpO2 (\%) & 90 & 87 & 95 & 99 \\
\hline $\mathrm{HB}(\mathrm{g} d \mathrm{~d})$ & 12.2 & 12,6 & 12.2 & 12,5 \\
\hline Ht (\%) & 38.4 & 41.1 & 40.2 & 36,8 \\
\hline D-dimer (ng/ml) & 2400 & & & 500 \\
\hline Troponine (ng/l) & $<50$ & $<50$ & & \\
\hline NT-proBNP (pg/ml) & & 24,478 & 9871 & 1449 \\
\hline Creatinine & 102 & 167 & 78 & 120 \\
\hline $\mathrm{cGFR} \mathrm{ml/min}$ & 57 & 35 & 74.88 & \\
\hline Urea & 6.1 & 8.9 & & \\
\hline $\mathrm{K}(\mathrm{mmol} / \mathrm{l}$ & 5.5 & 4 & & 5 \\
\hline Sodium (mmol/l) & 137 & 138 & & 140 \\
\hline Iron (mkmol/l) & & 11,8 & & 26,5 \\
\hline Bilirubin total $(\mathrm{mkmol} / \mathrm{l})$ & & 36 & & 28,4 \\
\hline Bilirubin direct (mkmol/l) & & 14.9 & & \\
\hline Homosystein $(\mathrm{mkmol} / \mathrm{l})$ & & & 28.1 & 31,4 \\
\hline Prothrombin G20210A & & & $\mathrm{G} / \mathrm{G}$ & \\
\hline Factor V Leiden F5 & & & $\mathrm{G} / \mathrm{G}$ & \\
\hline
\end{tabular}

HR, heart rate; BP, blood pressure; SpO2, peripheral capillary oxygen saturation; Hb, hemoglobin; Ht, hematocrit; NT-proBNP, N-terminal pro-brain natriuretic peptide; cGFR, calculated glomerular filtration rate; G/G, homozygous. 


\section{Discussion}

Deep venous thrombosis is frequently observed in patients with chronic heart failure and, of note, development of $\mathrm{PE}$ in patients with $\mathrm{CHF}$ is associated with very high mortality rate $[1,2]$.

Unfortunately, clinical evaluation of CHF patients with suspected acute PE is challenging since these diseases share several symptoms and signs such as dyspnea. However, the presence of well-known risk factors for DVT such as chronic venous insufficiency (CVI) with varicose vein (CEAP Class C2) [5], smoking, and hyper-homocysteinemia [2] should suggest a PE diagnosis. Our patient had CHF and all those risk factors for developing DVT (as witnessed by extensive DVT from popliteal, femoral, iliac via IVC up to the right atrium) and, probably, PE. Moreover, he had severe biventricular dysfunction with reduced myocardial function, clinical instability, which, taken together, appear to enhance the DVT risk associated due to decompensation of CHF. Unfortunately, in those patients in whom CHF becomes an acute event, symptoms of heart failure (e.g., dyspnea, cardiogenic shock, elevated jugular venous pressure), might act as confounding elements able to hide a DVT [4]. In fact, the previous diagnosis of $\mathrm{CHF}$ and the HF-related symptoms have probably played a role in delaying the correct diagnosis while the patient had several accesses to ER. Blood evaluation of CHF patients with suspected acute PE often does not help in obtaining a diagnosis since abnormal Ddimer, troponin, BNP and NT-proBNP might be measured in $\mathrm{CHF}$ patients. Thus, it should be suggested that patients with $\mathrm{CHF}$, abnormal cardiac biomarkers and findings of new or worsened right ventricle function should undergo further evaluation for PE. The proposed diagnostic and treatment strategy of PE in patient with CHF is shown in Figure 3. This diagnostic algorhytm foresees that CHF patients with suspected PE and with level of NT pro-BNP $>1905 \mathrm{pg} / \mathrm{ml}$ should be screened by using Wells score. Then, the most useful diagnostic tool is considered bedside transthoracic echocardiography (TTE). In a highly unstable patient, echocardiographic evidence of RV dysfunction is sufficient to prompt immediate reperfusion without further testing. This decision may be strengthened by the (rare) visualization of right-heart thrombi [6-9]. Ancillary bedside imaging tests include

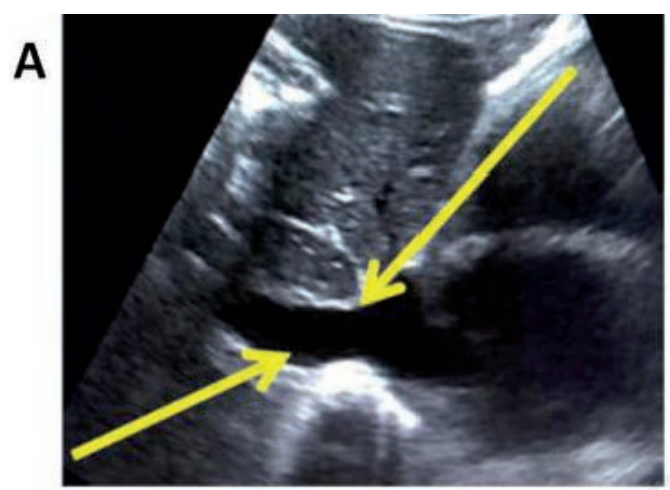

B
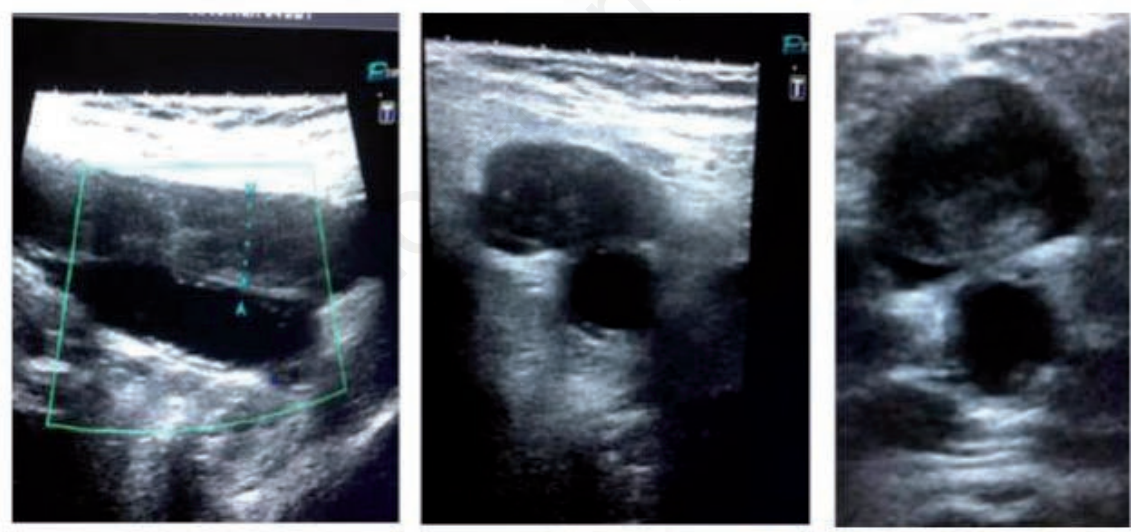

C
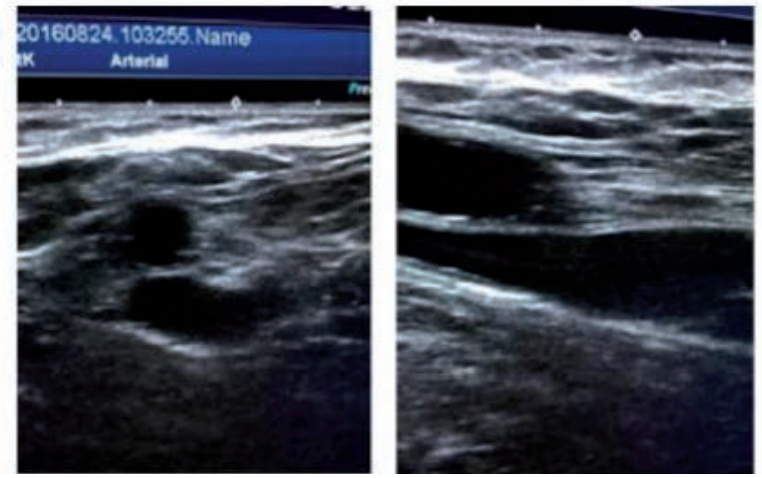

Figure 2. A) Ultrasound examination showed the complete resolution of IVCT (arrows). B) Ultrasound examination demonstrated thrombosis of the popliteal and deep femoral veins to the level of the femoral-iliac segments. C) Ultrasound examination after 2 months of treatment showed the absence of ultrasound signs of thrombosis of lower extremities. 


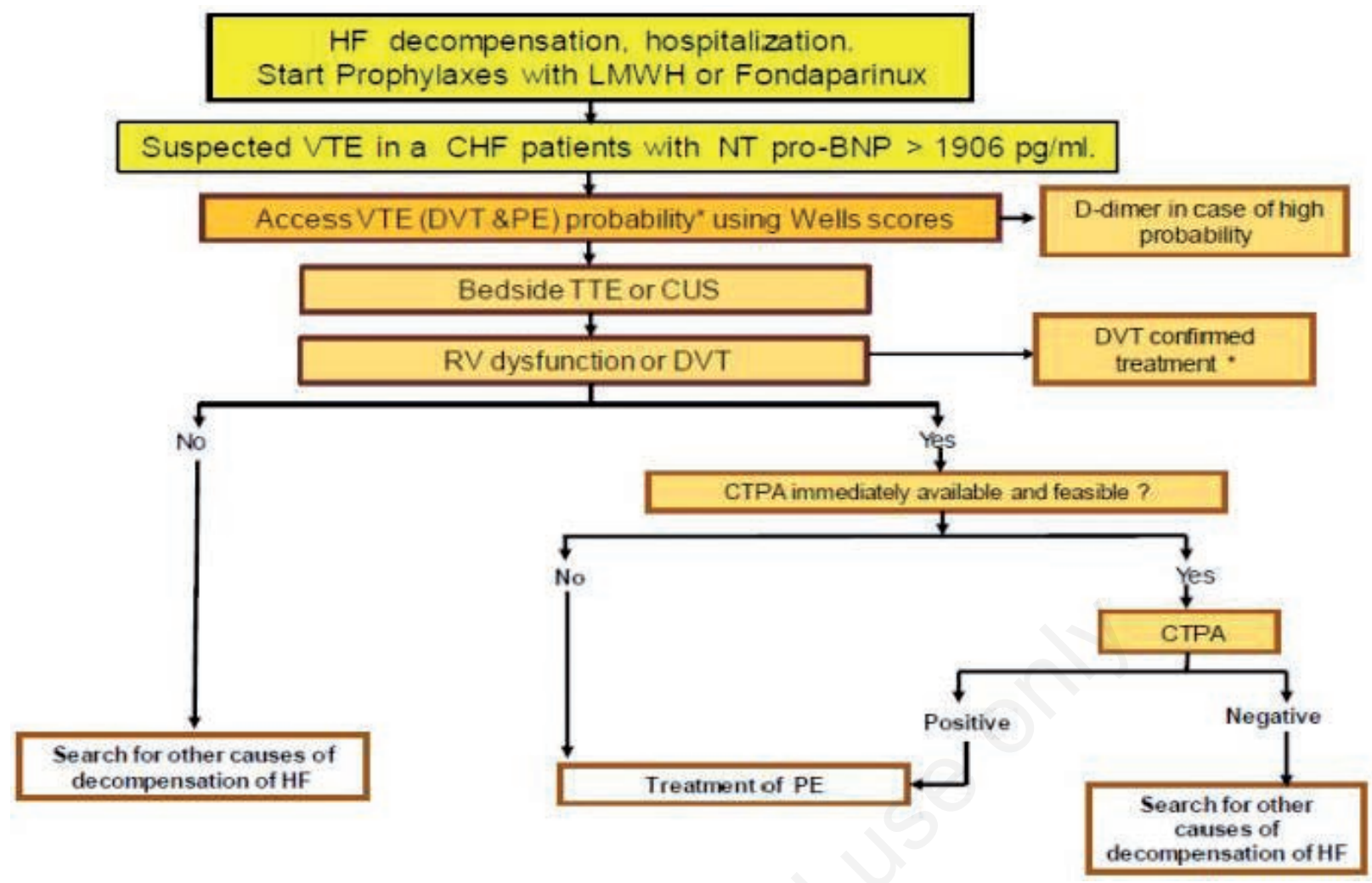

Figure 3. Proposed diagnostic and treatment algorithm for CHF patients with suspected pulmonary embolism.

transoesophageal echocardiography, which, if available, may allow direct visualization of thrombi in the pulmonary artery and its main branches, and bedside CUS, which can detect proximal DVT. In this report, TTE showed dilatation of both ventricle chambers, of right atrium, and, more important for the diagnosis, the "rare" presence of a thrombus in the right atrial cavity, close to the entering of the vena cava inferior. Moreover, US evaluation of veins showed a huge thrombosis of the popliteal, femoral and inferior vena cava. Taken together, these findings permitted to start antithombotic treatment and to avoid CT pulmonary angiography.

In conclusion, in this report we illustrate a rare case of extensive DVT in patient with CHF and PE, involving the lower extremities veins, the IVC up and the right atrium, poorly described in literature and indicate how imaging obtained by echocardiogram and peripheral ultrasound have an important role as early diagnostic tools.

\section{References.}

1. Ota S, Yamada N, Tsuji A, et al. Incidence and clinical predictors of deep vein thrombosis in patients hospitalized with heart failure in Japan. Circ J 2009;73:1513-17.

2. Mebazaa A, Spiro TE, Büller H, et al. Predicting the risk of venous thromboembolism in patients hospitalized with heart failure. Circulation 2014;130:410-8.
3. Bolorunduro O, Olatunde S, Singh A, et al. Lower extremity deep vein thrombosis is associated with mortality among patients hospitalized with congestive heart failure: Results from the Agency for Healthcare Research and Quality's Nationwide Inpatient Sample (1998-2007). J Vasc Med Surg 2013;1:4.

4. Darze ES, Latado AL, Guimarães AG, et al. Acute pulmonary embolism is an independent predictor of adverse events in severe decompensated heart failure patients. Chest 2007;131:1838-43-

5. Bergan JJ, Schmid-Schonbein GW, Coleridge Smith PD, et al. Chronic venous disease. N Engl J Med 2006;355:488-98.

6. Torbicki A, Perrier A, Konstantinides S, et al. Guidelines on the diagnosis and management of acute pulmonary embolism: the task force for the diagnosis and management of acute pulmonary embolism of the European Society of Cardiology (ESC). Eur Heart J 2008;29: 2276-315.

7. Torbicki A, Galie N, Covezzoli A, et al. Right heart thrombi in pulmonary embolism: results from the International Cooperative Pulmonary Embolism Registry. J Am Coll Cardiol 2003;41:2245-51.

8. Koc M, Kostrubiec M, Elikowski W, et al. Outcome of patients with right heart thrombi: the Right Heart Thrombi European Registry. Eur Respir J 2016;47:869-75.

9. Pierre-Justin G, Pierard LA. Management of mobile right heart thrombi: a prospective series. Int J Cardiol 2005;99:381-8. 